\title{
Articles
}

\section{Epidemiology of Type I diabetes mellitus in Switzerland: steep rise in incidence in under 5 year old children in the past decade}

\author{
E.J. Schoenle ${ }^{1}$, M. Lang-Muritano ${ }^{1}$, S. Gschwend ${ }^{2}$, J. Laimbacher ${ }^{3}$, P.E. Mullis ${ }^{4}$, T. Torresani ${ }^{1}$, \\ A. Biason-Lauber ${ }^{1}$, L. Molinari ${ }^{1}$ \\ ${ }^{1}$ University Children's Hospital, Zurich, Switzerland \\ ${ }^{2}$ Children's Hospital Luzern, Switzerland \\ ${ }^{3}$ Children's Hospital St. Gallen, Switzerland \\ ${ }^{4}$ University Children's Hospital, Berne, Switzerland
}

\section{Abstract}

Aims/hypothesis. In this nationwide prospective study we wanted to verify the trend of increasing diabetes incidence data from our earlier retrospective analysis of the military registry of Swiss men.

Subjects and methods. The data collection of newly diagnosed children in Switzerland at an age younger than 15 years started in 1991. The countrywide survey used a small questionnaire which was sent back to the study centre. The questionnaire was anonymous and contained: hospital of diagnosis, initials, sex, birth date, date of diagnosis, residence, country of citizenship, and responsible physician. General data on the population were taken from publications of the Swiss Federal Statistical Office.

Results. A total of 941 children below the age of 15 years with newly diagnosed Type I (insulin-dependent) diabetes mellitus were collected (434 girls, 507 boys). The incidence in children aged 0 to 14 years rose significantly between 1991 and 1999 with a yearly average increase of $5.1 \%$. In the age group 0 to 4 years a more than four-fold increase in incidence from $2.4 / 100000$ per year to $10.5 / 100000$ per year $(p=0.0002)$ was recorded, whereas the age-specific incidence in the 5 to 9-year-old and 10 to 14-year-old children did not change during the data collection period. The incidence was significantly higher in boys than in girls, whereas no difference was found between rural and urban populations.

Conclusion/interpretation. The incidence of Type I diabetes is rising in children living in Switzerland but only the youngest age group of under 5 years of age is affected showing a large annual average increase of $23.8 \%$. [Diabetologia (2001) 44: 286-289]

Keywords Epidemiology, incidence, Type I diabetes, children, Switzerland, childhood diabetes.
In a previous study [1] we reported the epidemiology of Type I (insulin-dependent) diabetes mellitus in Switzerland in 12 cohorts of Swiss men born between 1948 and 1972. We found an increase from 4.5 (earliest cohort) to 7.2 (latest cohort) per 100000 a year at an age younger than 15 years [1]. In 1991 a countrywide prospective study had been launched to learn more about the current epidemiology of Type I dia-

Received: 7 September 2000 and in revised form: 25 October 2000

Corresponding author: E.J. Schoenle, MD, Department of Paediatric Endocrinology \& Diabetology, University Children's Hospital, Steinwiesstrasse 75, CH-8032 Zurich, Switzerland betes in Swiss children. Switzerland is a small country (area $41300 \mathrm{~km}^{2}$ ) in the middle of Europe providing a good opportunity to carry out a nationwide study. In many countries worldwide prospective studies on the incidence of childhood diabetes have shown an increase in incidence in the past few decades [2-5]. Prospective data collection and evaluation are important for learning more about the possible dynamics in the epidemiology of Type I diabetes. The studies are usually done in children up to 15 years of age mainly because it is easier to collect data as children worldwide are mostly sent to hospitals after diagnosis for initiation of therapy and in contrast to diagnosing diabetes in adults, diagnosing diabetes in children is more clear and there are no doubts when distinguishing their type of diabetes. 
In this study we wanted to verify the trend of increasing incidence data from our own retrospective study and compare it with studies in other countries.

\section{Subjects and methods}

The prospective collection of data of newly diagnosed children younger than 15 years of age was started in Switzerland in 1991. The whole country participated. In Switzerland, children with newly diagnosed Type I diabetes are almost always sent to paediatric hospitals for initial therapy. Therefore all Swiss hospitals with a paediatric unit were invited to complete a small questionnaire which was then sent back to the study centre at the Children's University Hospital in Zurich. The questionnaire was anonymous and when completed contained the following information: hospital of diagnosis, initials, sex, date of birth, date of diagnosis, residence, country of citizenship, responsible physician. In certain years, not all data were received from all centres therefore the data had to be corrected for the missing region. Size, age distribution and ethnic composition of the Swiss population is continuously updated and published by the Swiss Federal Statistical Office [6]. The total population of Switzerland is 7.2 million people of which $19.4 \%$ are not Swiss citizens [6]. Only data from patients with verified diagnosis (discharge records) were used in the study. The second source of ascertainment was from the local offices of The Swiss Diabetes Association, a lay organization, that was asked twice to give initials, birth date, sex and living place of their members below 16 years of age. As a result $91.7 \%$ (query 1995) and $91.3 \%$ (query 2000) of those children who were members of the diabetes associations were identified in the survey. The study therefore shows a high ascertainment by a second source ensuring the countrywide epidemiological data was valid and representative.

In addition we have been collecting retrospective data from the Swiss Army Registry, providing a population of 1131744 Swiss men born between 1948 and 1972 with 2043 cases of Type I diabetes (manuscript in preparation). The methodology has been published earlier [1]. The high (96.6\%) ascertainment rate of our retrospective data [1] allows a comparison with our prospective study. The data of the children younger than the age of 5 years were compared with data from the prospective study of the same age group excluding girls and all children with foreign citizenship.

Regression analysis and analysis of covariance were used to assess the significance of the period (nine years) dependent change in incidence and of age dependent sex differences, respectively. A $p$-value of less than 0.05 was considered statistically significant.

\section{Results}

Between 1991 and 1999 a total number of 941 children (434 girls and 507 boys) below 15 years of age with newly diagnosed Type I diabetes have been reported to the study centre.

The annual incidence of the population investigated, children below younger than 15 years of age, and the age-specific incidence of children aged 0 to 4 years are shown in Figure 1. The incidence varies of course from year to year but with a significant

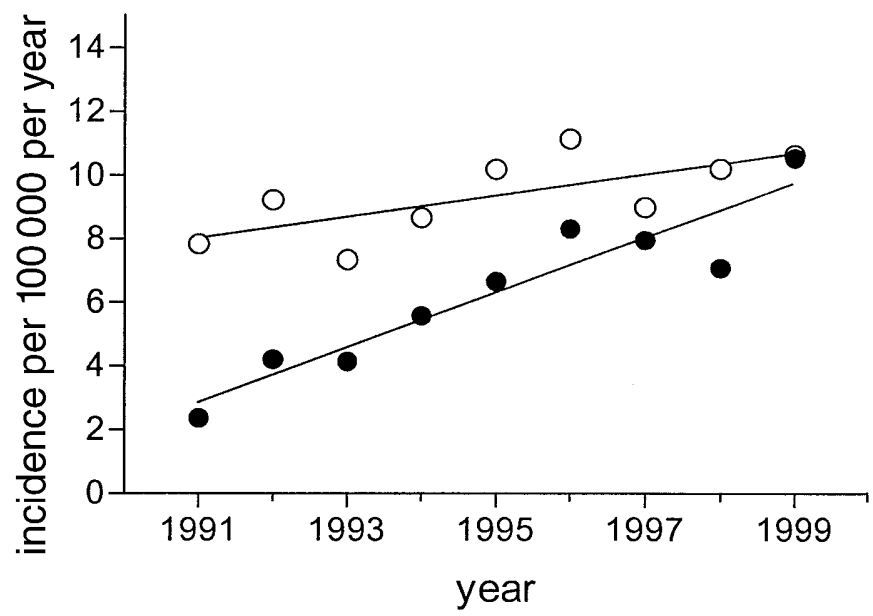

Fig. 1. Age-specific incidence of Type I diabetes mellitus in children aged 0 to 14 years $(\bigcirc)$ and 0 to 4 years $(\bigcirc)$ in Switzerland between 1991 and 1999. The solid line shows the average rate of change, which was significant in both age groups: 0 to 14 years: slope $=0.33 \pm 0.13(p=0.03)$ and 0 to 4 years: slope $=0.88 \pm 0.13(p=0.0002)$

$(p=0.03)$ increase between 1991 and 1999 from 7.8/ 100000 a year to $10.6 / 100000$ in the whole population, representing an annual average increase of $5.1 \%$. In the age group 0 to 4 years, we found a surprisingly steep, more than four-fold increase in incidence from 2.4/100000 a year to 10.5/100000 a year $(p=0.0002)$. This corresponds to an average yearly age-specific increase of $23.8 \%$. In contrast there was no significant change in incidence of the age groups of 5 to 9 years $(8.4 / 100000$ a year, $p=0.91)$ and 10 to 14 years (12.0/100000 a year, $p=0.92)$, meaning that between 1991 and 1999 only the youngest age group contributed to the overall increase of incidence.

Our data of the past decade in all age groups do not show any seasonality in the manifestation of Type I diabetes nor any variation of Type I diabetes diagnosis dependent on month of birth. In addition there was no difference $(p=0.2)$ in the incidence between rural and urban populations (results not shown).

A consistently higher incidence in boys than in girls was found. The difference in incidence between the boys and girls was significant (analysis of covariance) for the entire study population $(p=0.007)$ as well as for the age groups of 0 to 4 years $(p<0.0001)$ and 10 to 14 years $(p<0.05)$, whereas it was not different in the age group of 5 to 9 years $(p=0.55)$. The rate of the rise in incidence was the same between boys and girls.

The incidence rate of Type I diabetes in the youngest age group from the $1950 \mathrm{~s}$ is shown in Figure 2. As described, we have retrospective data available for Swiss men born between 1948 and 1972, allowing us to compare the age group below age 5 of the prospective study (1991-1999) with the retrospective study of 


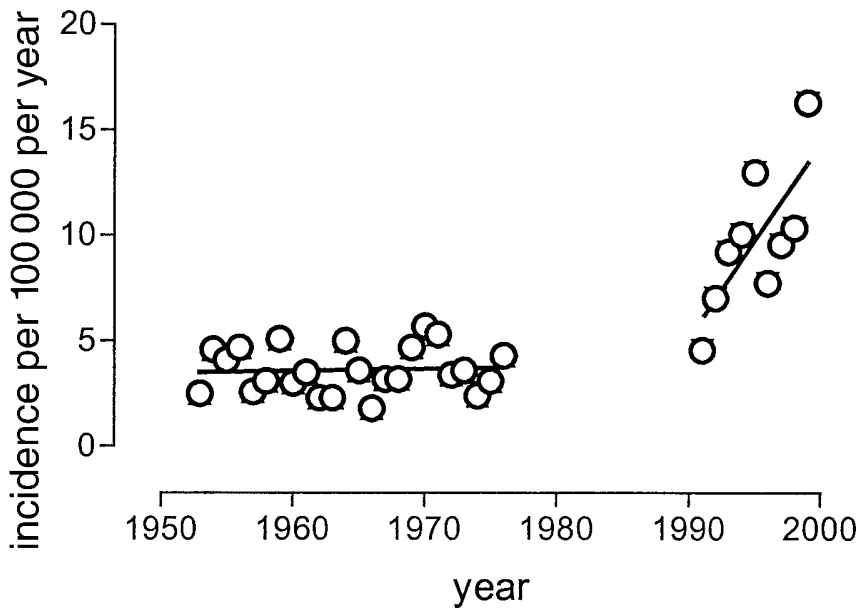

Fig. 2. Incidence of Type I diabetes mellitus in Swiss boys aged 0 to 4 years. The solid line shows the average rate of change in incidence, which was significant between 1991 and 1999 (slope $=0.92 \pm 0.31 ; p=0.02$ ) but not between 1953 and 1976 (slope $=0.01 \pm 0.03, p=0.78)$

the Swiss Military registry (1953-1976). There is, however, still a gap of 15 years in between. The population of the prospective study had to be adjusted for men with Swiss citizenship, because in the Swiss military registry only Swiss men are recorded. The agespecific incidence in the 0 to 4 year old boys was constant in the 24 years between 1953 and 1976 at an average incidence of $3.6 \pm 1.1($ mean \pm SD) $/ 100000$ a year without significant change $(p=0.8$, Fig. 2$)$. This was in pronounced contrast to the 9 years between 1991 and 1999, when the incidence in this population rose significantly $(p=0.02)$ from $4.5 / 100000$ in 1991 to $16.4 / 100000$ in 1999.

\section{Discussion}

As in most recent studies concerning epidemiology of Type I diabetes [2-5] our prospective data clearly show that the incidence in children and adolescents also rises significantly in Switzerland. The actual incidence of 10.5/100000 a year in children younger than 15 years of age is markedly higher than in our first retrospective evaluation with 4.5/100000 in 1965 and $7.8 / 100000$ in 1987 respectively [1]. This represents an annual increase of $5.1 \%$ between 1991 and 1999 compared with $3.3 \%$ between 1965 and 1987.

The rise in the incidence of Type I diabetes in the past decade is solely due to the contribution of the youngest age group (under 5 years of age). We find an extraordinary fourfold age-specific increase in the age group of 0 to 4 years between 1991 and 1999, in boys and girls and which was not influenced by ethnic background. For comparison, our retrospective data [1] show an unchanging incidence in this age group back to the earliest data available in 1953. The sharp rise in incidence beginning in 1992 is yet to be explained. Similar pronounced findings in the youngest age group have been found in some countries $[3,7$, 8] but not in others [5]. An annual increase of $11 \%$ was reported in this age group in the UK within 10 years [7] compared with the considerably higher annual average increase rate of $23.8 \%$ in the children younger than 5 years old in Switzerland. The incidence in the older age groups of 5 to 9 years and 10 to 14 years did not show any trend between 1991 and 1999, again in agreement with the findings in the UK [7]. These results show a true increase of Type I diabetes in children in Switzerland and not a shift to an earlier age of diagnosis. Whether this tendency will influence the incidence of Type I diabetes after childhood by means of a decreasing incidence rate in these birth-cohorts in the future as it has been shown in our earlier retrospective study [1] is very well possible, but could only be determined in a prospective study of Type I diabetes in adults.

Compared with studies in other countries we also found a higher incidence in boys than in girls in the population investigated with a pronounced difference in the youngest age group ( 0 to 4 years) and the oldest age group (10 to 14 years), but only a trend in the 5 to 9-year-old children. Again this corresponds with data from previous studies $[7,8]$ although we show a higher incidence in boys in the youngest age group.

In contrast to findings of other surveys, our data do not show any dependence on the month of birth of children with Type I diabetes nor do they show seasonal variation at diagnosis. Seasonality has been shown [9] but the effect does not show the same strength everywhere [10].

It is very difficult and very speculative to explain our finding of the high rise of Type I diabetes in young children in the past decade after its stability over at least 40 years. An obvious explanation, although difficult to prove, would be the influence of an exogenous factor triggering the islet cell destruction in genetically prone children. As there was not such an impressive obvious change in the daily lifestyle or eating behaviour in the past ten years than before, other triggers like viral infections of any kind or vaccination programme modifications as discussed elsewhere $[4,7,8]$ are at present speculative.

In conclusion, epidemiology of Type I diabetes in Switzerland is characterized by a high rise in the incidence in youngest age group of children younger than 5 years of age in the past decade.

Acknowledgements. The authors are indebted to the following persons for contributing in the collection of patients' data: H. E. Gnehm (Aarau), M. Wopmann, U. Lässer (Baden), J. Girard, U. Zumsteg (Basel), G. P. Ramelli, F. Taminelli (Bellinzona), C. Aebi, K. Zurbrügg (Biel), J. Lütschg (Bruderholz), H. Fricker (Chur), J.-M. Dubuis (Geneva), R. Maibach (Ilanz), G. Theintz, A. Maret (Lausanne), L. Buetti (Locarno), M. Gianninazzi (Lugano), G. Schubiger (Luzern), B. Regazzoni 
(Mendrisio), R. Haller (Münsterlingen), R. Bienentreu, N. Brunner (Samedan), A. Tschumi (Schaffhausen), G. Délèze, N. de Kalbermatten (Sion), U. Hunziker, E. Nützi (Winterthur), H. U. Meyer (Pompaples), U. Bühlmann (Zurich), and the local Diabetes Associations of Zurich, Luzern, Chur, St. Gallen, Basel, Schaffhausen, Aarau, Neuchatel, Geneva, Bellinzona, Sion, Delemont and Lausanne for their help in case ascertainment. This study was supported by the Swiss National Science Foundation, grants 3200-029863, 3200-039427 and 32-52724.97 to E. J. Schoenle.

\section{References}

1. Schoenle EJ, Molinari L, Bagot M, Semadeni S, Wiesendanger M (1994) Epidemiology of IDDM in Switzerland: Increasing incidence rate and rural-urban differences in Swiss men born 1948-1972. Diabetes Care 17: 955-960

2. Green A, Gale EAM, Patterson CC, for the EURODIAB ACE Study Group (1992) Incidence on childhood-onset insulin-dependent diabetes mellitus: the EURODIAB ACE study. Lancet 339: 905-909

3. EURODIAB ACE Study Group (2000) Variation trends in incidence of childhood diabetes in Europe. The Lancet 355 : 873-876
4. Tuomilehto J, Karvonen M, Pitkäniemi J et al. (1999) Record-high incidence of Type I (insulin-dependent) diabetes mellitus in Finnish children. Diabetologia 42: 655-660

5. Schober E, Schneider U, Waldhör T, Tuomilehto J, Austrian Diabetes Incidence Study Group (1995) Increasing incidence of IDDM in Austrian Children: a nationwide study 1979-1993. Diabetes Care 18: 1280-1283

6. Statistisches Jahrbuch der Schweiz. Bundesamt fuer Statistik, Bern, Verlag Neue Zürcher Zeitung 2000

7. Gardner SG, Bingley PG, Satwell PA, Weeks S, Gale EAM (1997) Rising incidence of insulin dependent diabetes in children aged under 5 years in the Oxford region: time trend analysis BMJ 315: 713-717

8. Karvonen M, Pitkäniemi J, Tuomilehto J, the Finnish childhood registry group (1999) The onset age of Type I diabetes in Finnish children has become younger. Diabetes Care 22: 1066-1070

9. Lévi-Marchal C, Patterson C, Green A, the EURODIAB ACE study group (1995) Variation by age group and seasonality at diagnosis of childhood IDDM in Europe. Diabetologia 38: 823-830

10. Karvonen M, Jantti V, Muntoni S et al. (1998) Comparison of the seasonal pattern in the clinical onset of IDDM in Finland and Sardinia. Diabetes Care 21: 1101-1109 